\title{
Forensische Andrologie
}

\section{Wann ist der Penis zu krumm für Sex?}

In Strafverfahren um strittige sexuelle Handlungen werden Ärzte nicht nur mit der Untersuchung des weiblichen, sondern auch des männlichen Genitals beauftragt, etwa zur Erfassung genitaler Verletzungen und zur Spurenasservierung. Mancher sieht bereits eine neue Spezialdisziplin "Forensische Andrologie" am Horizont. Die Fertilitätsdiagnostik könnte ebenfalls in deren Aufgabenfeld fallen. Ein weiterer forensischandrologischer Klassiker ist die Begutachtung der Penisanatomie als fragliches Penetrationshindernis.

Ausgangspunkt eines Begutachtungsauftrages an den Rechtsmediziner und Urologen PD Dr. Knut Albrecht, Hannover, war folgende Einlassung eines Rechtsanwalts: „Mein Mandant kann den angeschuldigten und erzwungenen $\mathrm{Ge}$ schlechtsverkehr nicht vollzogen haben, da er seit Jahren unter einer Verkrümmung des Penis leidet“.

\section{Der Deviationsgrad ist entscheidend}

Da er immer wieder mit Fragestellungen zur Penisdeviation (Infobox) konfrontiert wurde, ging Albrecht zusammen mit seinen Kollegen der Sache systematisch auf den Grund. Dazu untersuchten die Ärzte 30 Männer, die wegen einer Induratio penis plastica (IPP) die Urologische Poliklinik der Medizinischen Hochschu- le Hannover $(\mathrm{MHH})$ aufsuchten und befragten sie zu ihrer Sexualität. Die Männer waren zwischen 33 und 70 Jahre alt, der Altersdurchschnitt lag bei 60 Jahren.

22 hatten eine dorsale Deviation, drei eine ventrale Deviation, auch „Wasserhahndeformität“ genannt, und fünf eine Kombination aus einer lateralen mit entweder einer dorsalen oder ventralen Deviation. Die Ausprägung der Deviation lag bei der dorsalen Variante zwischen $40^{\circ}$ und $90^{\circ}$, bei der ventralen bei maximal $40^{\circ}$ und bei der lateralen bei $45-60^{\circ}$.

Die Befragung der Herren ergab, dass denjenigen mit einem dorsalen Deviationsgrad von $80^{\circ}$ oder mehr eine vaginale Penetration nicht möglich ist. Auch eine laterale Deviation ab $60^{\circ}$ ist demnach ein generelles Penetrationshindernis. Sechs
Das „gute Stück“ wird bei manchen Rechtsfragen auf Form und Funktion hin gründlich untersucht. 